\title{
Superficial Vein Thrombosis as the Initial Manifestation of Protein S Deficiency in an Adolescent with Narcolepsy
}

\author{
Su-Hyun Han, MD ${ }^{1,2}$, Sang-Ahm Lee, MD ${ }^{1}$ \\ ${ }^{1}$ Department of Neurology, Asan Medical Center, University of Ulsan College of Medicine, Seoul, Korea \\ ${ }^{2}$ Department of Neurology, Chung-Ang University College of Medicine, Seoul, Korea
}

\begin{abstract}
Protein S (PS) deficiency and narcolepsy have a similar pathomechanism in view of autoimmunity, and they can develop following an infection. However, there is no previous report of a patient with narcolepsy associated with PS deficiency and the relationship between these two diseases has not yet been investigated. PS deficiency may lead to coagulopathies, which can present as superficial or deep vein thrombosis and embolism. Therefore, here we describe a case of an 18-year-old man with narcolepsy who presented with superficial vein thrombosis associated with PS deficiency. Our case suggested that the development of PS deficiency and narcolepsy can be interrelated with each other.

Sleep Med Res 2016;7(1):39-41
\end{abstract}

Key Words Narcolepsy, Protein S deficiency, Superficial vein thrombosis.

Received: October 19, 2015 Revised: November 23, 2015 Accepted: December 7, 2015

\section{Correspondence}

Sang-Ahm Lee, MD Department of Neurology,

Asan Medical Center, University of Ulsan College of Medicine, 88 Olympic-ro 43-gil, Songpa-gu, Seoul 05505, Korea

Tel $+82-2-3010-3445$

Fax +82-2-474-4691

E-mailssalee@amc.seoul.kr

\section{INTRODUCTION}

Protein S (PS) is a vitamin K-dependent plasma protein; a cofactor to activated protein C for inactivation of factors Va and VIIIa, resulting in reduced thrombin generation. It enhances the profibrinolytic effects of activated protein C. ${ }^{1}$ Clinically, patients with PS deficiency are at increased risk for venous thromboembolic disease, occasional arterial thrombosis, etc. ${ }^{2}$ PS deficiency may be either hereditary or acquired, ${ }^{1}$ and its acquired causes are seen in acquired illness like liver disease, disseminated intravascular coagulation (DIC), therapy with L-asparaginase and coumarin, etc. ${ }^{1}$ Autoimmune PS deficiency can develop following an infection. ${ }^{3}$ Recent developments in narcolepsy research also support the hypothesis of narcolepsy being an immune-mediated disease. Therefore, we suggest that both these diseases may be interrelated with each other in view of autoimmunity. However, there is no previous report of a patient with narcolepsy associated with PS deficiency. Here, we describe a case of an 18-year-old man with narcolepsy who presented with superficial vein thrombosis (SVT) associated with PS deficiency.

\section{CASE REPORT}

An 18-year-old man with narcolepsy presented with pain and redness of the left lower limb for five days after long-distance travel on long-haul flights, for 14 hours in economy class. He did not provide any history of varicose veins, previous thromboembolic episodes, recent surgery, bleeding disorder, or malignancy. Also, he did not have history of H1N1 infection and vaccination. He had a body mass index of $20.8 \mathrm{~kg} / \mathrm{m}^{2}$, which was normal for his age.

He had been suffering from hypersomnia since 15 years of age and he denied any history of cataplexy. Overnight polysomnography with multiple sleep latency test performed at the age of 16 years showed a mean sleep latency of $2.5 \mathrm{~min}$ and four episodes of sleep-onset REM pe- 


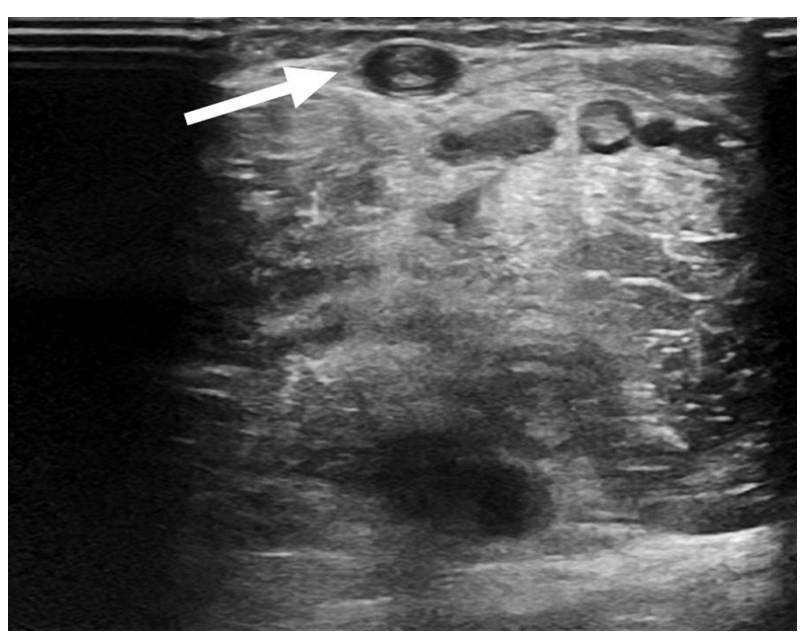

Fig. 1. Ultrasonography of the left ankle shows intraluminal acute thrombosis in the small saphenous vein (white arrow).

riods (SOREMPs) during five naps. The diagnosis of narcolepsy type 2 was made, ${ }^{4}$ for which he was receiving modafinil 200 $\mathrm{mg}$. After being prescribed the medicine, hypersomnia was significantly improved and he had a relatively good quality of life with stable daily activities.

On examination, he had a swollen left lower leg, and there was no other significant finding on systemic examination, including genital ulcer, skin lesion and eye inflammation. Peripheral arterial pulses were normal. Radiographs of both legs were normal. Ultrasonography revealed acute SVT in the left small saphenous vein (Fig. 1). Laboratory investigations revealed normal total and differential cell counts. Antinuclear factor, antiphospholipid antibodies, lupus anticoagulant and Venereal Disease Research Lavaratory titers were negative. His coagulation profile was normal except for the isolated abnormality of PS deficiency; total PS activity level was $41 \%$ and functional PS activity level was 30\%. Therefore, we made the diagnosis of SVT associated with PS deficiency in this patient with narcolepsy. This patient was managed with thrombectomy and supportive management including non-steroidal anti-inflammatory drugs and compression stockings.

\section{DISCUSSION}

We reported PS deficiency in an adolescent with narcolepsy type 2. He was diagnosed with narcolepsy type 2 at the age of 16 years, according to the International Classification of Sleep Disorders-3 (mean sleep latency $2.5 \mathrm{~min}$; SOREMPs 4 ), ${ }^{4}$ and his coagulation profile showed an isolated abnormality of PS deficiency. SVT was confirmed by Doppler ultrasonography. We found no evidence of other acquired or inherited thrombophilia. We excluded Behcet disease, neoplasia, and Buerger disease through medical history and follow-up. In our patient with narcolepsy, PS deficiency as well as long-distance travel on long-haul flights may have contributed to SVT.

Congenital PS deficiency was first reported in $1984^{5}$ and has subsequently been described in numerous families. ${ }^{6}$ Acquired deficiency of PS is observed in several pathological states which may be associated with an increased thrombotic risk. ${ }^{1}$ Autoimmune PS deficiency can develop following an infection, leading to devastating thrombotic manifestations, especially in children. ${ }^{3}$ PS deficiency in this patient is more likely to be acquired than inherited, because there was no family history of coagulopathy. Additionally other acquired causes including liver disease, DIC etc., were excluded through medical history and laboratory investigations. Therefore, we speculated that autoimmune mechanism may have contributed to PS deficiency in our patient.

A particularly interesting finding was that he had narcolepsy. Narcolepsy is characterized by hypersomnia, cataplexy etc. ${ }^{7} \mathrm{Al}-$ though the disease was first described towards the end of the 19th century, the underlying pathomechanisms were described only within the last decade. ${ }^{8}$ The exact mechanisms of autoimmunity are poorly understood, but an increase in the incidence of narcolepsy after H1N1 infection and vaccination supported the hypothesis of an immune-mediated pathogenesis of narcolepsy. The generation of an experimental animal model of autoimmune narcolepsy could suggest the mechanism of autoimmune attack against hypocretin-producing cells. ${ }^{8}$ Individuals affected by narcolepsy present with not only sleep problems, but they also have substantially higher rates of medical (hypercholesterolemia, diseases of the digestive system, heart diseases etc.) and psychiatric diseases (e.g., major depressive disorder, bipolar disorder etc.) than the general population. ${ }^{9}$ Comorbidities are represented by endocrine, neurological and musculoskeletal disorders. ${ }^{10}$ These results clearly illustrate the complexity of medical history and vulnerability of individuals with narcolepsy. Although there is no previous report of narcolepsy associated with PS deficiency and the relationship between these two diseases has not yet been investigated, there is an ongoing study which is recruiting the patient groups that have coagulopathy and narcolepsy (http://www.ehealthme.com/cs/ thrombosis/narcolepsy/). Therefore, we suggest that these two diseases may be interrelated with each other in view of autoimmunity, and PS deficiency can be one of the medical conditions associated with narcolepsy.

We could make the diagnosis of SVT associated with PS deficiency in this adolescent with narcolepsy. It cannot be ruled out that PS deficiency may be only comorbidity in our narcolepsy patient because the relationship between these two diseases has not yet been investigated. However, both diseases have a similar pathomechanism in view of autoimmunity. Our case suggested that the development of PS deficiency and narcolepsy can be interrelated with each other. Additionally, we indicated that further study exploring the relationship between narcolepsy and PS deficiency is needed. This case report can serve as the starting point for further studies to evaluate the relationship be- 
tween PS deficiency and narcolepsy.

\section{Acknowledgments}

This report was supported by the Asan Medical Center in 2015.

\section{Conflicts of Interest}

The authors have no financial conflicts of interest.

\section{REFERENCES}

1. D’Angelo A, Viganò D’Angelo S. Protein S deficiency. Haematologica 2008;93:498-501.

2. Gupta PK, Ahmed RP, Bhattacharyya M, Kannan M, Biswas A, Kalra V, et al. Protein C system defects in Indian children with thrombosis. Ann Hematol 2005;84:85-8.

3. D’Angelo A, Della Valle P, Crippa L, Pattarini E, Grimaldi LM, Viganò D'Angelo S. Brief report: autoimmune protein S deficiency in a boy with severe thromboembolic disease. N Engl J Med 1993;328:1753-7.

4. Ito E, Inoue Y. [The International Classification of Sleep Disorders, third edition. American Academy of Sleep Medicine. Includes bibliographies and index]. Nihon Rinsho 2015;73:916-23.
5. Comp PC, Nixon RR, Cooper MR, Esmon CT. Familial protein S deficiency is associated with recurrent thrombosis. J Clin Invest 1984;74: 2082-8.

6. Mateo J, Oliver A, Borrell M, Sala N, Fontcuberta J. Laboratory evaluation and clinical characteristics of 2,132 consecutive unselected patients with venous thromboembolism--results of the Spanish Multicentric Study on Thrombophilia (EMET-Study). Thromb Haemost 1997;77: 444-51.

7. Klein G, Burghaus L, Vaillant M, Pieri V, Fink GR, Diederich N. Dysautonomia in narcolepsy: evidence by questionnaire assessment. J Clin Neurol 2014;10:314-9.

8. Partinen M, Kornum BR, Plazzi G, Jennum P, Julkunen I, Vaarala O. Narcolepsy as an autoimmune disease: the role of H1N1 infection and vaccination. Lancet Neurol 2014;13:600-13.

9. Ohayon MM. Narcolepsy is complicated by high medical and psychiatric comorbidities: a comparison with the general population. Sleep Med 2013;14:488-92.

10. Jennum P, Ibsen R, Knudsen S, Kjellberg J. Comorbidity and mortality of narcolepsy: a controlled retro- and prospective national study. Sleep 2013;36:835-40 